\title{
Noncoding RNAs and their epitranscriptomic influences in cancer
}

\author{
Duncan Ayers*,1,2 \\ ${ }^{1}$ Centre for Molecular Medicine \& Biobanking, University of Malta, Msida, Malta \\ ${ }^{2}$ Faculty of Biology, Medicine \& Health, University of Manchester, Manchester, UK \\ *Author for correspondence: Duncan.Ayers@um.edu.mt
}
"In conclusion, the continuing advances in analytical technologies are paving the way to uncover ever more aspects of the dark genome, as has already occurred with the discovery of the spectrum of ncRNA families"

First draft submitted: 26 July 2018; Accepted for publication: 1 August 2018; Published online: 16 October 2018

Since the initial discovery of the DNA molecular structure by Crick and Watson [1], scientists in such a field became ever more fascinated by the intriguing complexity of the human genome and the roles DNA provided in harnessing the genetic data locked within its double-stranded helices. In this respect, up until the turn of the millennium, all intronic DNA sequences were essentially considered as 'junk' and merely providing a 'scaffolding' template for the far more important exonic DNA sequences. However, this dogma was rapidly deconstructed particularly with the discovery of the RNA interference (RNAi) pathway discovered by Fire and Mello in 1998 [2], with this consequently leading to the identification of the major roles played by microRNAs (miRNAs) in RNAi [3]. These major discoveries highlighted the prospect that the noncoding regions of the DNA helix, and consequently its corresponding RNA transcript, possess far more functional and regulatory roles within all biological entities carrying such a precious life-enabling blue-print.

In essence, most of the novel roles portrayed by noncoding RNAs (ncRNAs) is of a gene regulatory nature, particularly by epitranscriptomic measures, to ensure inhibition of the translation process of mRNA into the designated protein/s from the individual gene of interest [4]. Ultimately, such novel and effective pathways provide an extra level of complexity and control on the vast spectrum of cellular physiological processes occurring in a continuous manner, both in healthy and in diseased states. Presently, the level of knowledge on the facets of the differing ncRNA families and their gene regulatory roles is constantly enhanced due to the expanded scientific interest in such a field, most importantly due to its massive potential in providing novel theranostic measures that can be applied to all human disease conditions having dysregulated genomic undertones, such as cancer. However, from the multiple ncRNA families depicted in the last 18 years, the two families with the most clinical value would be the miRNA and long noncoding RNA (lncRNA) families.

The human miRNA family comprises 2693 members, according to the latest miRBase (release 22) [5-7]. Each mature miRNA comprises a 19-25 nucleotide-long RNA duplex, with the active strand having the capacity to bind to the target transcript $3^{\prime}$ UTR in order to effect post-transcriptional mRNA regulation, mainly by obstructing ribosomal activity [8]. In the context of cancer, miRNAs can act as tumor suppressors or as oncogenic miRNAs (oncomiRs) [9].

A primary example of tumor suppressor miRNAs would be hsa-miR-34a-5p as it was identified to be involved in the regulation of multiple human cancer model characteristics such as metastasis, chemoresistance properties and cellular invasiveness [10].

In contrast, there exist a profusion of oncomiRs that pose a severe threat to the cancer patient through further accelerating tumor progression by exacerbating a myriad of aggressive tumor phenotypes. Taking chemoresistance properties as a typical example, we have compiled a comprehensive compendium of all known miRNAs (and IncRNAs) that directly/indirectly influence cancer chemoresistance properties within most human cancer conditions [11]. Together with the above-mentioned example, there exists a whole plethora of other tumor phenotypes that are affected by miRNA activity. 
The lncRNA family currently comprises over 58,000 members, all of which are a minimum of 200 nucleotides in length and this entire ncRNA family comprises $68 \%$ of the human transcriptome [12]. Initially discovered by the Functional Annotation of Mammalian cDNA (FANTOM) project earlier in this millennium, the lncRNA family members are the result of RNA polymerase II/III processing and are consequently subjected to transcriptional editing prior to forming a stable secondary/tertiary structure in which they exert their functional roles [13]. The interesting feature of lncRNAs is that their functional roles could be implemented at the transcriptional or posttranscriptional stages and can either comprise gene regulatory roles or as enhancers of gene expression [13]. In addition, individual lncRNAs can exert their influence either on separate regions of their own host chromosome (cis-action) or on regions of completely different chromosomes (trans-action) [13].

Within the context of cancer, of all the lncRNA family members, two individual lncRNAs stand out from the crowd.

The HOTAIR is one of the most recognized and studied IncRNAs having oncogenic properties in multiple human tumors, acting as an oncogenic catalyst for a spectrum of tumor phenotypes including genomic stability, metastasis, anti-apoptotic behavior and chemoresistance [14]. Similarly, the MALAT1 has been implicated as a highly influential oncogenic lncRNA in a long list of human cancer models [11,15].

Apart from the well-described oncogenic IncRNAs briefly mentioned above, there exist other novel lncRNAs having emerging functional roles within specific tumors. Most notably, the recent study by Leucci and colleagues identified an individual lncRNA, SAMMSON, to be highly co-expressed with the melanoma-specific oncogene MITF [16]. In addition, knockdown of the SAMMSON IncRNA led to a reduction in viability of melanoma cells and also increased melanoma sensitivity to MAPK-targeted chemotherapeutic measures [16].

The discovery of such influential miRNA and lncRNA families certainly leads one to recognize the degree of genomic and epitranscriptomic interplay that exists in order to induce specific cellular phenotypes, including within the tumor cellular context. However, to add insult to injury, there also exists a high level of regulatory or proexpression interplay between individual/networks of miRNAs and lncRNAs simultaneously and with other ncRNA families, in order to exert niche phenotypes within the target tissues. The latest study conducted by Kleaveland and colleagues highlights this notion perfectly, by elucidating an intricate network of several ncRNA families acting together in order to regulate mammalian brain neuronal activities [17]. In this study, the investigators elucidated that lncRNA Cyrano expression induced miR-7 degradation led to uninhibited expression of the circRNA Cdr1as which is widely involved in regulating neuronal interactions [17].

The degree of complexity illustrated in the above novel ncRNA families and their molecular interplays, no matter how intricate, still essentially boils down to basic epitranscriptomic activities - or in other terms - basic interactions with the RNA transcript. Consequently, it is of no wonder that the level of interest and academic/industrial research into epitranscriptomics has increased drastically over the past decade. However, as with all other medical research niches, the need to network, share similar scientific interests and also collaborate with other passionate researchers in the same field of expertise is key in providing a catalyst for additional scientific knowledge and also future theranostics based on such novel findings. Addressing this challenge within the EU alone, there exists a European Commission Institute (Cooperation in Science and Technology [COST]; www.cost.eu) that provides specific COST Actions that have funding to facilitate networking and collaborative research efforts for furthering knowledge in such specific niches. In this respect, one of the current COST Actions, initiated in 2017, is the European Epitranscriptomic Network (EPITRAN - CA16120). This action allows for research groups from 27 participating European (and neighboring) countries to gather and share their interdisciplinary skills and knowledge to further the European scientific community's insight on epitranscriptomic fields such as RNA modifications, ncRNA influences and other research fields falling within the realm of epitranscriptomics [18]. Should the reader be interested in participating within the EPITRAN Network, they are strongly advised to contact the author or contact the EPITRAN directly through the COST website portal for assistance.

In conclusion, the continuing advances in analytical technologies are paving the way to uncover ever more aspects of the dark genome, as has already occurred with the discovery of the spectrum of ncRNA families, a few of which have been briefly discussed above. Increase in research, collaborative efforts and innovation technologies could only enhance the prospect of utilizing the revealed knowledge, as yet hidden within the dark genome, for the development of novel diagnostic and therapeutic measures against life-threatening conditions such as cancer. The author is highly confident that this hidden realm will provide the key to such medical advancements in the not-too-distant future and that will directly benefit patients in a rapid and affordable manner in the clinical management of their genomic-based condition. 


\section{Financial \& competing interests disclosure}

The author has no relevant affiliations or financial involvement with any organization or entity with a financial interest in or financial conflict with the subject matter or materials discussed in the manuscript. This includes employment, consultancies, honoraria, stock ownership or options, expert testimony, grants or patents received or pending, or royalties.

No writing assistance was utilized in the production of this manuscript.

\section{References}

1. Watson JD, Crick FH. Genetical implications of the structure of deoxyribonucleic acid. Nature 171(4361), 964-967 (1953).

2. Fire A, Xu S, Montgomery MK, Kostas SA, Driver SE, Mello CC. Potent and specific genetic interference by double-stranded RNA in Caenorhabditis elegans. Nature 391(6669), 806-811 (1998).

3. Lagos-Quintana M, Rauhut R, Lendeckel W, Tuschl T. Identification of novel genes coding for small expressed RNAs. Science 294(5543), 853-858 (2001).

4. Huang B, Zhang R. Regulatory noncoding RNAs: revolutionizing the RNA world. Mol. Biol. Rep. 41(6), 3915-3923 (2014).

5. Griffiths-Jones S. The microRNA registry. Nucleic Acids Res. 32(Database issue), D109-D111 (2004).

6. Griffiths-Jones S, Grocock RJ, van Dongen S, Bateman A, Enright AJ. miRBase: microRNA sequences, targets and gene nomenclature. Nucleic Acids Res. 34(Database issue), D140-D144 (2006).

7. Griffiths-Jones S, Saini HK, van Dongen S, Enright AJ. miRBase: tools for microRNA genomics. Nucleic Acids Res. 36(Database issue), D154-D158 (2008).

8. Rolle K, Piwecka M, Belter A et al. The sequence and structure determine the function of mature human miRNAs. PLoS ONE 11(3), e0151246 (2016).

9. Carta A, Chetcuti R, Ayers D. An introspective update on the influence of miRNAs in breast carcinoma and neuroblastoma chemoresistance. Genet. Res. Int. 2014, 743050 (2014).

10. Farooqi AA, Tabassum S, Ahmad A. MicroRNA-34a: a versatile regulator of myriads of targets in different cancers. Int. J. Mol. Sci. 18(10), doi:10.3390/ijms18102089 (2017).

11. Ayers, D, Vandesompele J. Influence of microRNAs and long noncoding RNAs in cancer chemoresistance. Genes 8(3), doi:10.3390/genes8030095 (2017).

12. Sanchez Calle A, Kawamura Y, Yamamoto Y, Takeshita F, Ochiya T. Emerging roles of long noncoding RNA in cancer. Cancer Sci. 109(7), 2093-2100 (2018).

13. Ayers D. Long noncoding RNAs: novel emergent biomarkers for cancer diagnostics. J. Cancer Res. Treat. J. Cancer Res. Treat. 1(2), 31-35 (2013).

14. Tang Q, Hann SS. HOTAIR: an oncogenic long noncoding RNA in human cancer. Cell. Physiol. Biochem. 47(3), 893-913 (2018).

15. Zhao M, Wang S, Li Q, Ji Q, Guo P, Liu X. MALAT1: a long noncoding RNA highly associated with human cancers. Oncol. Lett. 16(1), 19-26 (2018).

16. Leucci E, Vendramin R, Spinazzi M et al. Melanoma addiction to the long noncoding RNA SAMMSON. Nature 531(7595), 518-522 (2016).

17. Kleaveland B, Shi CY, Stefano J, Bartel DP. A network of noncoding regulatory RNAs acts in the mammalian brain. Cell 174(2), 350-362 e17 (2018).

18. Jantsch MF, Quattrone A, O'Connell M et al. Positioning Europe for the EPITRANSCRIPTOMICS challenge. RNA Biol. 9, 1-3 (2018). 
